\title{
Desenvolvimento do Pensamento Computacional por Meio da Colaboração: uma revisão sistemática da literatura
}

\author{
Computational Thinking Development through Collaboration: a systematic review
}

Ângelo Magno de Jesus

Universidade Cruzeiro do Sul

(UNICSUL)/ Instituto Federal de

Minas Gerais (IFMG)

angelo.jesus@ifmg.edu.br

\author{
Ismar Frango Silveira \\ Universidade Cruzeiro do Sul \\ (UNICSUL) \\ ismar.silveira@cruzeirodosul.edu.br
}

\author{
Wagner Barbosa de Lima Palanch \\ Universidade Cruzeiro do Sul \\ (UNICSUL) \\ wagner.palanch@cruzeirodosul.edu. \\ $\underline{b r}$
}

\begin{abstract}
Resumo
A inclusão do Pensamento Computacional nas salas de aula pode trazer grandes avanços para a educação, uma vez que através dele, estudantes podem exercitar diversas habilidades como a capacidade de resolução de problemas complexos, raciocínio lógico, abstração entre outras. A colaboração também representa um aspecto chave para o aprendizado. As interações sociais entre alunos advindas de métodos de Aprendizagem Colaborativa podem auxiliar no desenvolvimento cognitivo de diferentes formas. Neste contexto, este artigo descreve uma revisão sistemática da literatura a respeito do aprendizado do Pensamento Computacional associado a Aprendizagem Colaborativa. O objetivo deste estudo foi o de identificar como abordagens e métodos colaborativos de aprendizagem estão sendo empregados em atividades para o desenvolvimento do Pensamento Computacional e quais são os problemas resolvidos e as lacunas encontradas nestes trabalhos.
\end{abstract}

Palavras-Chave: Colaboração, Pensamento Computacional, Revisão Sistemática.

\begin{abstract}
The inclusion of the Computational Thinking in classroom can bring great advances to education. Through Computational Thinking students can exercise logical reasoning, solve complex problems, deal with abstraction among other skills. Collaboration is also a key aspect of learning. Social interactions among students from collaborative learning methods could contribute to the cognitive development in different ways. In this context, this article describes a systematic review of the literature regarding the learning of Computational Thinking associated with Collaborative Learning. The main goal of this study was to identify how Collaborative Learning approaches are being used in the Computational Thinking development. This article also tries to identify solved problems and gaps on these studies.
\end{abstract}

Keywords: Collaboration, Computational Thinking, Systematic Review.

Cite as: de Jesus, A. M, Silveira, I. F. \& Palanch, W. B. de L. (2019). Computational Thinking Development through Collaboration: a systematic review (Desenvolvimento do Pensamento Computacional por Meio da Colaboração: uma revisão sistemática da literatura). Brazilian Journal of Computers in Education (Revista Brasileira de Informática na Educação - RBIE), 27(2), 69-90. DOI: 10.5753/RBIE.2019.27.02.69 


\section{Introdução}

Muitos são os desafios para se criar uma educação de qualidade, dentre estes, podemos encontrar o problema de tornar os estudantes mais autônomos na construção de seu próprio saber. É preciso que o ambiente escolar não ensine estudantes apenas a memorizar conceitos, mas os ensine a lidar com problemas complexos de diferentes domínios, uma vez que o conhecimento está em constante evolução e as informações mudam e se expandem a cada dia. Neste contexto, o Pensamento Computacional pode representar um ferramental poderoso para que estudantes possam compreender, traçar estratégias e propor soluções para questões provenientes de várias áreas do conhecimento como matemática, ciências, linguagens etc. No entanto, a inserção do Pensamento Computacional nas salas de aula ainda representa um desafio que precisa ser investigado exaustivamente. Desta forma, questiona-se "quais seriam as abordagens e métodos adequados para tornar o desenvolvimento do Pensamento Computacional, em estudantes, mais efetivo?". Vygotsky, conforme Moreira (2011), já ressaltava a importância das interações sociais no processo de aprendizagem. Segundo o autor, a aprendizagem ocorre primeiro nos processos mentais superiores (pensamento, linguagem e comportamento voluntário) que têm sua origem em processos sociais, desta forma o desenvolvimento cognitivo é a conversão de relações sociais em funções mentais. Neste contexto, a Aprendizagem Colaborativa pode ser uma poderosa aliada para o ensino de diferentes disciplinas incluindo o Pensamento Computacional, uma vez que abordagens colaborativas de aprendizado focam no fortalecimento das interações sociais entre alunos, e por vezes entre alunos-professor, com a finalidade de construir o conhecimento mutuamente. Através deste paradigma, estudantes tem a possibilidade de aprender novos conceitos com outros alunos, conhecer suas próprias potencialidades e limitações, respeitar as diferenças, entender múltiplos pontos de vista sobre um assunto entre outros.

Este trabalho propõe realizar uma revisão sistemática da literatura com o objetivo de buscar entender como a Aprendizagem Colaborativa está sendo utilizada para melhorar, em diferentes aspectos, o desenvolvimento de habilidades do Pensamento Computacional em estudantes. Para tal, foram coletadas publicações de duas bases de dados importantes para o campo do Pensamento Computacional, a base de dados da Association for Computing Machinery (ACM) e a base de periódicos da Coordenação de Aperfeiçoamento de Pessoal de Nível Superior (CAPES). Após a seleção, os estudos foram mapeados e classificados de acordo com diferentes aspectos, incluindo modelos de colaboração, atividades desenvolvidas entre outros. Como consequência, procuramos fazer um levantamento de quais pontos já estão sendo atacados por trabalhos recentes na literatura e quais aspectos ainda necessitam de uma maior exploração por parte de pesquisadores da área.

\section{Embasamento Teórico}

Esta seção discute conceitos básicos que foram considerados para o desenvolvimento da análise sistemática da literatura.

\subsection{Pensamento Computacional}

O conceito de Pensamento Computacional foi popularizado por Wing (2006) como uma habilidade fundamental para todos em todos os lugares. Apesar disso, a ideia não se originou pela autora, Papert (1993) no final dos anos 60, já descrevia experiências que almejavam ensinar conceitos de ciência e matemática para crianças por meio da programação de computadores através da linguagem Logo. Além disso, o termo Pensamento Computacional já tinha sido 
utilizado por Papert (1980), nesta obra o autor discute como os computadores poderiam afetar a forma com que as pessoas pensam e aprendem, especialmente no contexto do ensino infantil. Conforme Wing (2006), o Pensamento Computacional pode auxiliar na capacidade analítica de crianças para ler, escrever e para aritmética. Trata-se de um conjunto de ferramentas mentais fundamentadas na Ciência da Computação que auxiliam na resolução de problemas de diferentes áreas do conhecimento. A autora também destaca que o Pensamento Computacional combina e complementa as formas de pensamento utilizadas da Matemática e da Engenharia, a primeira devido a fundamentação de todas ciências na matemática e a segunda devido ao Pensamento Computacional envolver a idealização de sistemas que interagem ou têm relação com o mundo real.

CSTA\&ISTE (2011) descrevem o Pensamento Computacional como uma abordagem de solução de problemas que fortalece a integração de tecnologias digitais com ideias humanas. Esta abordagem possui foco nas habilidades relacionadas ao raciocino lógico, à criatividade e ao pensamento crítico, enquanto enfatiza formas de se organizar um problema de modo que um computador possa auxiliar em sua solução. Barr e Stephenson (2011) afirmam que o Pensamento Computacional pode ser entendido como uma metodologia de resolução de problemas que pode ser automatizada, transferida e aplicada em todos os assuntos. Os alunos não se tornam apenas usuários de ferramentas, mas construtores. Conforme os pesquisadores, os estudantes devem utilizar um conjunto de conceitos (como abstração, recursão e iteração) para processar e analisar dados e para criar artefatos reais ou virtuais. Os autores também apresentam uma série de atividades do Pensamento Computacional: coleta de dados, análise de dados, representação de dados, decomposição de problemas, abstração, algoritmos e procedimentos, automação, paralelização e simulação.

O Pensamento Computacional, conforme Barr e Stephenson (2011), apresenta os seguintes aspectos aplicáveis de valores, motivações, sentimentos, estereótipos e atitudes:

(1) Confiança em lidar com a complexidade;

(2) Persistência em trabalhar com problemas difíceis;

(3) A capacidade de lidar com a ambiguidade;

(4) A capacidade de lidar com problemas abertos;

(5) Deixar de lado as diferenças para trabalhar com os outros para alcançar um objetivo comum ou uma solução;

(6) Conhecer os pontos fortes e fracos de alguém ao se trabalhar com outras pessoas.

Cabe observar que os itens (5) e (6) estão relacionados diretamente ao Aprendizado Colaborativo. Além disso, Barr e Stephenson (2011) também propõem estratégias para beneficiar a experiência de aprendizado, que incluí a realização do trabalho em equipe entre os alunos, com uso explícito de:

(A) Decomposição - quebrar problemas em partes menores que podem ser mais facilmente resolvidas;

(B) Abstração - simplificar do concreto ao geral à medida que as soluções são desenvolvidas;

(C) Negociação - grupos dentro da equipe trabalham juntos para unir partes da solução como um todo;

(D) Construção de consenso - trabalhar para construir uma solidariedade coletiva por trás de uma idéia ou solução. 
Estas colocações trazem uma reflexão a respeito da aplicação do Aprendizado Colaborativo no desenvolvimento de habilidades do Pensamento Computacional. Cabe observar também que Papert, em suas obras, descreve possibilidades e experiências de colaboração no aprendizado, por meio da programação de computadores, em atividades construcionistas (PAPERT, 1980). O autor destaca o uso dos computadores como uma forma de aprendizes se engajarem em uma verdadeira colaboração intelectual (PAPERT, 1993, p.115). Definiremos a seguir o conceito de Aprendizagem Colaborativa.

\subsection{Aprendizagem Colaborativa}

Dillenbourg (1999) relata que Aprendizagem Colaborativa é amplamente definida como uma situação em que duas ou mais pessoas aprendem ou tentam aprender alguma coisa juntas. O autor argumenta que esta definição não é satisfatória, pois cada elemento desta definição pode ser interpretado de uma maneira diferente. Portanto, para Dillenbourg (1999) a Aprendizagem Colaborativa é descrita como uma situação na qual formas específicas de interação entre pessoas devem ocorrer. Uma preocupação geral é desenvolver formas de aumentar a probabilidade de ocorrer algum tipo de interação, pois, apesar destas interações possibilitarem o desencadeamento de mecanismos de aprendizagem, não há garantia de que as interações esperadas possam realmente ocorrer.

Para Smith e MacGregor (1992) a Aprendizagem Colaborativa é um termo abrangente para uma variedade de abordagens educacionais que envolvem esforço intelectual conjunto por estudantes, ou estudantes e professores juntos. Os autores argumentam que, normalmente, os alunos estão trabalhando em grupos para buscar mutuamente compreensão, soluções e significados, ou até mesmo criar um produto. Ainda conforme os autores, as atividades de Aprendizagem Colaborativa variam amplamente, mas a maioria se concentra na exploração ou aplicação do material do curso por parte dos alunos, e não simplesmente na apresentação ou na explicação por parte do professor.

Laal e Laal (2012) relatam que a Aprendizagem Colaborativa sugere uma maneira de lidar com pessoas que respeitam e destacam as habilidades e contribuições dos membros individuais do grupo. O princípio desta abordagem se baseia na construção de consenso através da cooperação dos membros do grupo, em contraste com a concorrência em que os indivíduos tentam ser melhores que outros membros. Portanto, existe um compartilhamento de autoridade e aceitação de responsabilidade entre os indivíduos para as ações dos grupos. Os autores também concluem que os principais elementos da Aprendizagem Colaborativa incluem: interdependência positiva, interação considerável, responsabilidade individual, habilidades sociais e processamento de grupo.

Cabe observar que as três definições apresentadas relatam, de alguma forma, que o ensino através do Aprendizado Colaborativo se distanciam do ensino tradicional instrucionista. Na definição de Dillenbourg (1999) existe a preocupação geral de desenvolver e aumentar a interação entre participantes. No ensino instrucionista esta preocupação não existe, uma vez que a interação é unidirecional de professor para alunos. Na definição de Smith e MacGregor (1992) esta distinção fica clara devido a exploração e aplicação do material por parte dos alunos. Por fim, Laal e Laal (2012) relatam que existe um compartilhamento de autoridade e construção de consenso, também contradizendo o ensino instrucionista tradicional no qual a autoridade está centralizada na figura do professor. Cabe ressaltar que o aprendizado colaborativo está fundamentado na epistemologia construtivista (Panitiz, 1999).

No que diz respeito ao estímulo de habilidades do Pensamento Computacional, o aprendizado colaborativo pode ser de grande interesse. Conforme o estudo apresentado por 
Gokhale (1995), o aprendizado colaborativo pode auxiliar no desenvolvimento do pensamento crítico e da habilidade de resolução de problemas, fatores extremamente importantes para o Pensamento Computacional.

\section{Metodologia}

O procedimento adotado para condução da revisão sistemática foi baseado no método utilizado por Barcelos (2014), porém com realizações de adaptações para atender o contexto e escopo deste estudo. Para realização deste trabalho, formulou-se primeiramente a seguinte questão de pesquisa "Como a Aprendizagem Colaborativa se manifesta em atividades que utilizam e estimulam o Pensamento Computacional?’. A partir desta questão base, foi definida a String de busca que foi estruturada no seguinte formato:

"computational thinking" AND (collab* OR cooper*)

Cabe ressaltar, que a string considerou as variações que poderiam surgir do termo "collaborative" para assim alcançar um maior conjunto de trabalhos. Como alguns autores ainda não diferenciam os termos Aprendizagem Colaborativa e Aprendizagem Cooperativa (PANITIZ, 1999), o termo "cooperative" também foi considerado na string de busca.

A pesquisa foi restringida para os últimos 5 anos, uma vez que se trata de uma área em constante evolução e se espera obter estudos atuais que considerem o conhecimento já produzido pelos trabalhos desenvolvidos anteriormente. A busca foi realizada em duas bases de dados importantes:

- Portal de Periódico da CAPES (CAPES, 2017): trata-se de um sítio que permite acesso a vários periódicos internacionais e nacionais em múltiplas áreas do conhecimento. Conforme (CAPES, 2017), o Portal de Periódicos da Capes oferece acesso a textos completos disponíveis em mais de 38 mil publicações periódicas, internacionais e nacionais, e a diversas bases de dados que reúnem desde referências e resumos de trabalhos acadêmicos e científicos até normas técnicas, patentes, teses e dissertações dentre outros tipos de materiais, cobrindo todas as áreas do conhecimento. Inclui também uma seleção de importantes fontes de informação científica e tecnológica de acesso gratuito na web.

- ACM (ACM, 2017): Por ter sido a instituição na qual o termo Pensamento Computacional se definiu e ganhou popularidade entre pesquisadores. Trata-se de uma base que agrega diversos artigos em variadas linhas de pesquisa da Ciência da Computação.

Os artigos foram filtrados primeiramente pelo resumo. Nesta etapa, foram eliminados artigos em que os conceitos de Colaboração e de Pensamento Computacional se apresentaram de forma muito superficial. Estudos que geraram dúvidas a respeito da profundidade em abordar os conceitos chave ou sobre sua contribuição para a questão de pesquisa foram filtrados posteriormente por meio de uma consulta ao texto integral do artigo. Também foram eliminados artigos com a palavra colaboração em outro sentido (ex. interação escola-universidade, colaboração entre instituições ou entre profissionais etc), capas de anais de conferência, projetos e propostas de pesquisas que não demonstram nenhum tipo de resultado, propostas de painéis e oficinas publicados em anais, keynotes de conferências que não apresentam texto com reflexões sobre o tema, proposta de sessões para congressos, informações sobre autores, biografias de autores, contra capas de revistas, notas dos editores, artigos que apresentam o Pensamento Computacional no sentido de algoritmos voltados para outras áreas da Ciência da Computação (como Otimização ou Inteligência Computacional). Também foram eliminados trabalhos que 
propunham atividades práticas, mas não demonstravam nenhum relato de realização destas atividades. Artigos que não possuíam uma seção de resumo ou que não continham informações suficientes nesta seção foram selecionados através do texto integral.

Uma vez que os artigos foram selecionados, com o objetivo de identificar tendências, avanços e limitações nesta linha de pesquisa, foi realizada um série de classificações. Apresentamos a seguir as principais.

\section{(A) Empírico/Conceitual}

Os estudos foram divididos nestas categorias a partir de uma abordagem semelhante a empregada por Barcelos (2014). Desta forma, os trabalhos foram classificados como Empíricos, pesquisas que apresentam experimentos empíricos sobre Pensamento Computacional e Colaboração, ou Conceituais, trabalhos que apresentam discussões teóricas sobre o tema, normalmente contendo argumentações baseadas em uma análise documental ou expressando opiniões baseadas em experiências. Estas discussões podem incluir a manifestação de um ponto de vista, mapeamento curricular e revisões da literatura.

Os trabalhos que se enquadraram na categoria Empírica também foram classificados de acordo com os itens (B), (C), (D), (E), (F) e (G) listados abaixo. Estudos que foram classificados como Conceituais foram também categorizados de acordo com o item $(\mathrm{H})$ no final da lista.

\section{(B) Público Alvo}

Esta categoria procurou identificar o público alvo dos estudos de acordo com o nível de educação formal em que os experimentos foram realizados.

\section{(C) Instrumentos de Pesquisa}

Neste item, buscamos identificar quais instrumentos de pesquisa foram utilizados para coleta e análise de dados dos trabalhos empíricos. Cabe ressaltar, que um trabalho pode ter utilizado mais de um instrumento de pesquisa. Por exemplo, um estudo pode ter utilizado entrevistas e observações para coletar dados e avaliar sua abordagem.

\section{(D) Modelo de Colaboração Realizada}

Diz respeito a como a colaboração foi tratada pelos pesquisadores. Envolve os métodos e abordagens utilizados para promover as interações sociais e consequentemente a colaboração entre os alunos para realização das atividades que compõem os experimentos. Neste contexto, podem haver trabalhos que estimulam a colaboração entre alunos, apenas os colocando para fazer uma atividade em grupo e os deixando livres para definir como o trabalho será realizado, outros podem focar em métodos mais bem estruturados, como a Programação em Par. As classes deste modelo foram levantadas durante a avaliação dos estudos e portanto, serão apresentadas na seção de resultados.

\section{(E) Modelo de Atividades Desenvolvidas}

Diz respeito a qual formato de atividade foi realizada em colaboração. Estas atividades podem envolver o desenvolvimento colaborativo de um jogo, o projeto colaborativo de robôs, solução de desafios etc. Esta classificação se relaciona a como (através de quais atividades) o Pensamento Computacional é inserido no Aprendizado Colaborativo. Assim como no item (D), as classes deste modelo foram levantadas durante a avaliação dos estudos e portanto, serão apresentadas na seção de resultados.

\section{(F) Artefatos Desenvolvidos}


A realização de atividades para promover o Pensamento Computacional, geralmente, envolve uma série de abordagens que podem ser empregadas para desenvolvimento de sistemas computacionais. Desta forma, as atividades aplicadas pelos estudos empíricos podem envolver a construção de diferentes artefatos para tornar a aprendizagem mais construcionista (Papert, 1993). Podem haver estudos em que os alunos devem desenvolver robôs, jogos, simulações e até mesmo artefatos mais abstratos, como a solução algorítmica para um problema de lógica. As classes desta categoria emergiram durante a análise dos estudos e serão apresentadas na seção de resultados.

\section{(G) Tecnologias Utilizadas nas Atividades}

Para desenvolvimento de artefatos, conforme destacado no item (F), ou mesmo para realização de práticas colaborativas para aprendizado do Pensamento Computacional, vários estudos podem adotar o uso de diferentes ferramentas tecnológicas. Esta categoria diz respeito a quais tecnologias foram aplicados para os alunos desenvolverem as atividades propostas nos estudos selecionados

\section{(H) Modelo de Trabalho Conceitual}

Assim como Barcelos (2014), trabalhos que se encaixaram na categoria conceitual foram classificados como (1) Ponto de Vista; (2) Revisão Sistemática e (3) Mapeamento Curricular.

\section{Resultados}

Como relatado anteriormente, as pesquisas foram realizadas nas bases de dados da CAPES e da ACM. A Tabela 1 mostra a quantidade de resultados encontrados em cada uma das bases antes e depois da seleção descrita na seção 3.

Tabela 1: Resultado da pesquisa antes e após a filtragem.

\begin{tabular}{|c|c|c|}
\hline Base & Antes da Seleção & Após Seleção \\
\hline CAPES & 464 & 28 \\
\hline ACM & 59 & 9 \\
\hline Total & 523 & 37 \\
\hline
\end{tabular}

Cabe informar que na busca realizada na base da CAPES encontramos resultados repetidos, que não necessariamente ocorreram em sequência. Foram notados pelo menos 11 repetições nos resultados. Além disso, três estudos foram eliminados por não estarem disponíveis para acesso na base de dados e não foram encontrados por meio de máquinas de busca para internet, apesar de apresentarem os requisitos para seleção no resumo. Os seguintes periódicos foram listados no resultado de busca da base da CAPES: ACM Transactions on Computing Education (TOCE); ACM Transactions on Computinig Education; Communications of the ACM; Computers \& Education; Computers \& Amp Education; Computers in Human Behavior; Education and Information Technologies; Interactive Technology and Smart Education; International Journal of Technology and Design Education; Journal of Educational Computing Research; Journal of Parallel and Distributed Computing; Journal of Science Education and Technology; Journal of Science Teacher Education; PLoS Computational 
Biology; Robotics and Autonomous Systems; Science and Children; Science Scope e Science Teacher. Apesar da base da CAPES ter apresentado vários periódicos da ACM em seus resultados, não foram encontradas interseções entre os resultados da busca nas duas bases.

A Figura 1 mostra um gráfico ilustrando a quantidade de artigos (dentre os selecionados) por ano de publicação. Como pode ser observado na figura, existe um movimento ascendente no número de artigos publicados a cada ano. Desta forma, com o decorrer do tempo há uma tendência de desenvolvimento de trabalhos sobre Aprendizado Colaborativo e Pensamento Computacional.

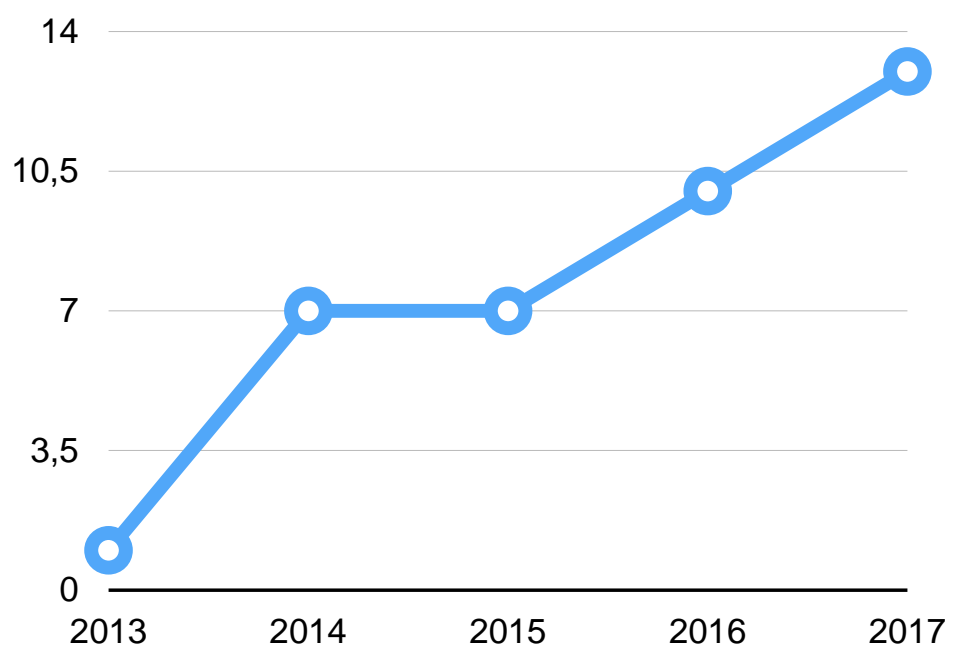

Figura 1: Gráfico representando a quantidade de trabalhos publicados por ano.

\section{(A) Empírico/Conceitual}

A partir da análise dos 37 estudos selecionados, foram identificados 34 trabalhos empíricos e apenas 3 trabalhos considerados como conceituais (Tabela 2). Estes dados evidenciam a escassez de trabalhos teóricos que relacionam colaboração com Pensamento Computacional. Observa-se, portanto, o longo caminho ainda a ser trilhado por pesquisadores da área, uma vez que menos de $10 \%$ dos trabalhos são de caráter conceitual.

Tabela 2: Trabalhos Empíricos e Conceituais.

\begin{tabular}{|c|c|c|}
\hline Tipo & $\begin{array}{c}\text { Quantidade de } \\
\text { Trabalhos }\end{array}$ & Porcentagem \\
\hline Empíricos & 34 & $91.9 \%$ \\
\hline Conceituais & 3 & $8.1 \%$ \\
\hline
\end{tabular}

\section{(B) Público Alvo}

A Tabela 3 mostra a divisão de trabalhos por categoria de ensino. Cabe observar que a maioria das pesquisas foca no Ensino Fundamental e no Ensino Superior respectivamente. A menor parcela de estudos, sem mencionar trabalhos mistos, se dedica aos docentes. Identificamos que apenas 2 trabalhos foram destinados ao público docente. Os trabalhos González e Muñoz-Repiso (2017) e Hsu e Wang (2017) apresentam atividades para o público de 
estudantes mas relatam de forma superficial que os experimentos na realidade foram realizados com docentes, por isso consideramos estes estudos focados para este último público.

Tabela 3: Trabalhos segmentados por categoria de ensino.

\begin{tabular}{|c|c|c|}
\hline Ensino & Trabalhos & Porcentagem \\
\hline $\begin{array}{l}\text { Educação } \\
\text { Primária }\end{array}$ & $\begin{array}{l}\text { Zhong et al. (2017); Falloon (2017); Zhong, Wang e Chen (2016); } \\
\text { Apostolellis et al. (2014); Harlow e Leak (2014), Mak (2014). }\end{array}$ & $17,65 \%$ \\
\hline $\begin{array}{l}\text { Ensino } \\
\text { Fundamental }\end{array}$ & $\begin{array}{l}\text { Thomas et al. (2017); Fronza, Ioini e Corral (2017); Buffum (2016); } \\
\text { Hartl et al. (2015); Ke e Im (2014); Wilkerson-Jerde (2014); Horn et al. } \\
\text { (2016). }\end{array}$ & $20,59 \%$ \\
\hline Ensino Médio & $\begin{array}{c}\text { Brady et al. (2017); Reynolds (2016); Caglar et al. (2015); Deitrick, } \\
\text { Wilkerson e Simoneau (2017); Fetaji et al. (2016). }\end{array}$ & $14,70 \%$ \\
\hline $\begin{array}{l}\text { Ensino } \\
\text { Superior }\end{array}$ & $\begin{array}{c}\text { Silapachote e Srisuphab (2017); Gestwicki e Mcnely (2016); } \\
\text { Frydenberg (2015); Goodman e Dekhtyar (2014); Hu e Shepherd } \\
\text { (2013); Pulimood, Pearson, Bates (2016); Burns et al. (2014); } \\
\text { Damaševicius et al. (2017). }\end{array}$ & $23,53 \%$ \\
\hline Docentes & Hsu e Wang (2017); González e Muñoz-Repiso (2017). & $5,88 \%$ \\
\hline $\begin{array}{l}\text { Ensino Médio } \\
\text { e Fundamental }\end{array}$ & $\begin{array}{l}\text { Tabel et al. (2017); Pellas e Peroutseas (2016); Papavlasopoulou } \\
\text { (2017); Thieme (2017); Berland; Davis e Smith (2015). }\end{array}$ & $14,71 \%$ \\
\hline $\begin{array}{l}\text { Ensino } \\
\text { Primário e } \\
\text { Ensino } \\
\text { Fundamental }\end{array}$ & Lee (2016). & $2,94 \%$ \\
\hline
\end{tabular}

\section{(C) Instrumentos de Pesquisa}

A relação de trabalhos por instrumentos de pesquisa pode ser observada na Tabela 4. Nesta relação, um trabalho pode possuir mais de um instrumento de pesquisa. Como exemplo, um trabalho pode ter sido considerado tanto no uso de questionários quanto de entrevistas. Cabe observar que a maioria dos estudos, focou no uso de questionários. Muitos estudos também utilizaram avaliações de aprendizagem e observações. A maioria dos trabalhos que utilizou avaliação de aprendizagem, o fizeram através de um pré-teste e um pós-teste. No entanto, Thomas et al. (2017) aplicaram um jornal para reflexão (um tipo de diário de bordo) que foi utilizado pelos participantes durante a realização dos experimentos. Cabe observar que não foram encontrados trabalhos que adotaram o índice de aprovação como instrumento de pesquisa.

Tabela 4: Instrumentos de Pesquisa Utilizados.

\begin{tabular}{|c|c|}
\hline Instrumento & Trabalhos \\
\hline Questionário & $\begin{array}{l}\text { Thomas et al. (2017); Fronza, Ioini e Corral (2017); Brady et al. (2017); Zhong et } \\
\text { al. (2017); Zhong, Wang e Chen (2016); Buffum (2016); Pellas e Peroutseas (2016); } \\
\text { Hartl et al. (2015); Frydenberg (2015); Goodman e Dekhtyar (2014); Horn et al. } \\
\text { (2016); Burns et al. (2014); Fetaji et al. (2016); }\end{array}$ \\
\hline $\begin{array}{l}\text { Avaliação de } \\
\text { aprendizagem - antes e } \\
\text { após intervenção }\end{array}$ & $\begin{array}{l}\text { Thomas et al. (2017); Fronza, Ioini e Corral (2017); Zhong et al. (2017); Zhong, } \\
\text { Wang e Chen (2016); Buffum (2016); Hartl et al. (2015); Caglar et al. (2015); } \\
\text { Wilkerson-Jerde (2014); Papavlasopoulou (2017); Pulimood, Pearson, Bates (2016); } \\
\text { Horn et al. (2016). }\end{array}$ \\
\hline
\end{tabular}




\begin{tabular}{|c|c|}
\hline Entrevista & $\begin{array}{l}\text { Fronza, Ioini e Corral (2017); Brady et al. (2017); Pellas e Peroutseas (2016); } \\
\text { Reynolds (2016); Caglar et al. (2015); Fetaji et al. (2016). }\end{array}$ \\
\hline Observação & $\begin{array}{l}\text { Thomas et al. (2017); Falloon (2017); Gestwicki e Mcnely (2016); Buffum (2016); } \\
\text { Reynolds (2016); Frydenberg (2015); Ke e Im (2014); Wilkerson-Jerde (2014); } \\
\text { Deitrick, Wilkerson e Simoneau (2017); Thieme (2017); Harlow e Leak (2014). }\end{array}$ \\
\hline Relatos & $\begin{array}{l}\text { Tabel et al. (2017); Hsu e Wang (2017); Brady et al. (2017); Mak (2014); González } \\
\text { e Muñoz-Repiso (2017); Lee (2016); Apostolellis et al. (2014). }\end{array}$ \\
\hline Verificação de notas & Silapachote e Srisuphab (2017); Hu e Shepherd (2013); Damaševicius et al. (2017). \\
\hline $\begin{array}{l}\text { Análise de Artefatos } \\
\text { produzidos }\end{array}$ & Fronza, Ioini e Corral (2017); Brady et al. (2017); Reynolds (2016); Ke e Im (2014), \\
\hline $\begin{array}{l}\text { Análise de registros de } \\
\text { logs ou de eyetracking }\end{array}$ & Berland; Davis e Smith (2015); Wilkerson-Jerde (2014); Papavlasopoulou (2017). \\
\hline
\end{tabular}

\section{(D) Modelos de Colaboração}

A definição de Estilo de Colaboração Realizada foi dividida nas seguintes classes:

1. Trabalho em Grupo: Trabalhos que relataram que as atividades colaborativas foram realizadas em grupos ou equipes sem utilizar abordagens mais estruturadas ou que não descreveram estruturalmente a abordagem de colaboração adotada. Geralmente, este tipo de estudo deixa a colaboração por autonomia do grupo de estudantes.

2. Programação em Par: Trabalhos que utilizaram a abordagem de Programação em Par tal como utilizada em Metodologias Ágeis de Desenvolvimento de Software, na qual se considera o papel do Piloto (programador) e Co-piloto (ajudante) e há circulação dos estudantes entre estes papéis.

3. Design Iterativo Colaborativo: Trabalhos em que equipes desenvolvem projetos normalmente de desenvolvimento de um produto completo (como, por exemplo, um jogo digital) em um ciclo iterativo. Nestes métodos, os grupos recebem feedback constantemente para realizar refinamentos no produto final. Cabe observar que esta classe pode abranger a Programação em Par, no entanto, os trabalhos desta categoria envolvem outras abordagens complementares a essa.

4. Design Colaborativo - outras abordagens: Assim como no item 3, trabalhos em que equipes desenvolvem projetos geralmente ligados ao desenvolvimento de um produto completo, no entanto, estas abordagens não envolvem processos iterativos nos quais os grupos precisam dar feedbacks constantes.

5. Solução Colaborativa de Problemas/Desafios: Trabalhos nos quais os experimentos propõem atividades em que alunos devem resolver em conjunto uma série de desafios e/ou problemas, típicos do Pensamento Computacional, propostos pelos professores ou tutores das atividades. Esta categoria esta associada ao Problem Based Collaborative Learning. Normalmente, os trabalhos classificados nesta categoria se assemelharam muito aos estudos classificados como "Trabalho em Grupo", uma vez que muitos estudos deixam a entender que os grupos de estudantes tiveram autonomia na colaboração.

6. Colaboração através de Ambiente On-line: Estudos que tratam do uso de ambientes on-line para colaboração. Nestas propostas a colaboração ocorre através da comunicação, compartilhamento de informações e troca de conhecimento através das ferramentas digitais fornecidas por estes ambientes. Desta forma, o Pensamento Computacional pode ser 
desenvolvido, de forma colaborativa, pelo compartilhamento de soluções (como algoritmos) e/ou discussões entre alunos sobre as mesmas no ambiente on-line.

7. Simulações Participativas: Trabalhos em que os estudantes colaboram através de uma "dramatização" coordenada. Neste sentido, os alunos devem se comportar e interagir de acordo com os papéis dos componentes de um sistema. A medida que a atividade avança, a sala de aula como um todo experimenta fenômenos e comportamentos de sistemas (BRADY et al., 2017). Neste contexto, o Pensamento Computacional é inserido por dramatizações por parte dos aprendizes que podem encenar, por meio de atuações em conjunto, o funcionamento de um sistema computacional, como o protocolo de envio de pacotes em uma rede de computadores por exemplo.

8. Sala de Aula Invertida: Estudos que utilizaram o método de Sala de Aula Invertida, em que o tempo de sala de aula é melhor aproveitado para realização de dinâmicas e discussões entre alunos sobre a matéria estudada ou experimentada fora da sala de aula. Neste modelo, o professor passa uma série de materiais on-line, como vídeos e textos, a respeito do Pensamento Computacional, para que o estudante absorva no período extraclasse. Durante o período de aula, as habilidades do Pensamento Computacional, vistas anteriormente, podem ser trabalhadas por meio de exercícios e atividades práticas (como programação de robôs, por exemplo) realizadas por equipes de alunos. A colaboração ocorre quando alunos podem auxiliar uns aos outros de acordo com o conteúdo que cada um adquiriu fora de sala de aula.

A Tabela 5 ilustra os trabalhos presentes em cada categoria. Podemos observar que a maioria dos estudos (38.24\%) se concentra em atividades que visam manter os alunos trabalhando em grupos deixando, assim, a colaboração por conta dos mesmos. Outra boa parcela de estudos (20.59\%) propõe o uso de Solução Colaborativa de uma Série de Problemas e Desafios. As outras abordagens de colaboração possuem representatividade menor que 15\% cada. Várias possibilidades de estratégias e métodos de Aprendizagem Colaborativa (incluindo métodos de origem no aprendizado de programação) precisam ser melhor explorados e estudados, por exemplo, o Dojo de Programação poderia ser aplicado por ser um método colaborativo, inclusivo e de aprendizado contínuo (Sato; Corbucci \& Bravo, 2008). Outro exemplo de abordagem que poderia ser melhor estudada seria a formação de equipes para competições e maratonas, a qual requer que estudantes tenham que colaborar e resolver conflitos. Esta atividade poderia ser mais explorada, uma vez que o fator competitivo entre times pode engajar os estudantes nas interações. Ainda neste contexto, apesar da abordagem da Sala de Aula Invertida estar ganhando popularidade no ensino, apenas o trabalho de Fetaji et al. (2016) apresenta este escopo de estudo.

Tabela 5: Modelo de Colaboração.

\begin{tabular}{|c|c|c|}
\hline $\begin{array}{c}\text { Atividade } \\
\text { Colaborativa }\end{array}$ & Trabalhos & Porcentagem \\
\hline $\begin{array}{c}\text { Trabalho em } \\
\text { Grupo }\end{array}$ & $\begin{array}{c}\text { Hsu e Wang (2017); Frydenberg (2015); Goodman e Dekhtyar } \\
\text { (2014); Mak (2014); González e Muñoz-Repiso (2017); } \\
\text { Papavlasopoulou (2017); Thieme (2017); Lee (2016); } \\
\text { Pulimood, Pearson, Bates (2016); Horn et al. (2016); Burns et } \\
\text { al. (2014); Harlow e Leak (2014); Apostolellis et al. (2014). }\end{array}$ & $38,24 \%$ \\
\hline $\begin{array}{c}\text { Programação } \\
\text { em par (com } \\
\text { troca de } \\
\text { piloto) }\end{array}$ & $\begin{array}{c}\text { Tabel et al. (2017); Zhong et al. (2017); Zhong, Wang e Chen } \\
\text { (2016); Buffum (2016); Hartl et al. (2015); }\end{array}$ & $14,71 \%$ \\
\hline
\end{tabular}




\begin{tabular}{|c|c|c|}
\hline $\begin{array}{l}\text { Design } \\
\text { Iterativo } \\
\text { Colaborativo }\end{array}$ & $\begin{array}{l}\text { Thomas et al. (2017); Fronza, Ioini e Corral (2017); Gestwicki } \\
\text { e Mcnely (2016); }\end{array}$ & $8,82 \%$ \\
\hline $\begin{array}{c}\text { Design } \\
\text { Colaborativo - } \\
\text { outras } \\
\text { abordagens }\end{array}$ & Reynolds (2016); Ke e Im (2014); Damaševicius et al. (2017). & $8,82 \%$ \\
\hline $\begin{array}{c}\text { Solução } \\
\text { Colaborativa } \\
\text { de Série de } \\
\text { Problemas/Des } \\
\text { afios/Atividad } \\
\text { es }\end{array}$ & $\begin{array}{l}\text { Silapachote e Srisuphab (2017); Falloon (2017); Pellas e } \\
\text { Peroutseas (2016); Berland; Davis e Smith (2015); Caglar et } \\
\text { al. (2015); Hu e Shepherd (2013); Deitrick, Wilkerson e } \\
\text { Simoneau (2017). }\end{array}$ & $20,59 \%$ \\
\hline $\begin{array}{l}\text { Ambiente On- } \\
\text { line } \\
\text { Colaborativo }\end{array}$ & Wilkerson-Jerde (2014). & $2,94 \%$ \\
\hline $\begin{array}{l}\text { Simulações } \\
\text { Participativas }\end{array}$ & Brady et al. (2017). & $2,94 \%$ \\
\hline $\begin{array}{l}\text { Sala de Aula } \\
\text { Invertida }\end{array}$ & Fetaji et al. (2016). & $2,94 \%$ \\
\hline
\end{tabular}

\section{(E) Modelos de Atividades Desenvolvidas} classes:

A classificação de Modelos de Atividades Desenvolvidas foi dividida nas seguintes

1. Projeto de Desenvolvimento de Jogos ou Animações Interativas: Atividades em que a equipe de alunos deve projetar e desenvolver colaborativamente um Jogo Digital ou um artefato similar a um jogo. São trabalhos que envolvem como foco principal a concepção de um produto final como resultado do projeto.

2. Projeto de Robótica: trabalhos que enfocam a construção, ou programação, de robôs ou artefatos relacionados a robótica através de kits e materiais eletrônicos. Cabe destacar que o objetivo dos trabalhos desta categoria também visa o desenvolvimento de um projeto completo seja de montagem de um robô (ou dispositivo robótico), seja de implementação de um algoritmo para o robô realizar alguma tarefa específica.

3. Projeto de Software - outros: estudos que propõem que alunos desenvolvam um software completo voltado para outras áreas do conhecimento.

4. Solução de Problemas ou Desafios Propostos: trabalhos nesta categoria focam na solução, por parte dos alunos, de uma série de desafios e problemas que foram propostos pelos responsáveis. Alguns trabalhos desta categoria envolveram, de alguma forma, a programação de pequenos jogos entre os desafios propostos, no entanto não envolveram o desenvolvimento de um projeto de jogo completo.

5. Atividade de Coleta e Análise de Dados: atividade em que alunos devem coletar e analisar dados para tirar conclusões sobre algum determinado assunto.

6. Oficina ou aula prática: atividades que propõem o ensino ou realização de oficinas ou aulas geralmente focadas em práticas para desenvolvimento do Pensamento Computacional. 
7. Jogar um Game: estudos em que as práticas eram realizadas de forma que os estudantes deveriam jogar um game, digital ou não, em conjunto.

É possível observar que algumas categorias desta classificação e da Classificação (F) "Modelos de Colaboração" possuem relações diretas. Entretanto, a criação de duas formas de classificação se faz necessária para se entender como as atividades de colaboração ocorrem em diferentes aspectos.

Fica claro a necessidade de mais trabalhos que enfoquem no desenvolvimento de Projetos de Robótica, uma vez que apenas $5.88 \%$ dos trabalhos foram encaixados nessa classe. Além disso, existem necessidade de trabalhos focados em atividades de coleta de dados e competições (como foi relatado anteriormente).

Tabela 6: Tipos de Atividades Realizadas.

\begin{tabular}{|c|c|c|}
\hline $\begin{array}{l}\text { Tipo de } \\
\text { Atividade }\end{array}$ & Trabalhos & Porcentagem \\
\hline $\begin{array}{c}\text { Projeto de } \\
\text { Desenvolvime } \\
\text { nto de Jogos } \\
\text { ou Animações } \\
\text { interativas }\end{array}$ & $\begin{array}{l}\text { Thomas et al. (2017); Tabel et al. (2017); Fronza, Ioini e } \\
\text { Corral (2017); Gestwicki e Mcnely (2016); Reynolds } \\
\text { (2016); Hartl et al. (2015); Frydenberg (2015); Ke e Im } \\
\text { (2014); Lee (2016); }\end{array}$ & $26,47 \%$ \\
\hline $\begin{array}{l}\text { Projeto de } \\
\text { Robótica }\end{array}$ & Damaševicius et al. (2017); Fetaji et al. (2016). & $5,88 \%$ \\
\hline $\begin{array}{l}\text { Projeto de } \\
\text { Software- } \\
\text { outros }\end{array}$ & $\begin{array}{l}\text { Pulimood, Pearson, Bates (2016); Goodman e Dekhtyar } \\
\text { (2014); }\end{array}$ & $5,88 \%$ \\
\hline $\begin{array}{l}\text { Solução de } \\
\text { Problemas ou } \\
\text { Desafios } \\
\text { Propostos }\end{array}$ & $\begin{array}{l}\text { Silapachote e Srisuphab (2017); Brady et al. (2017); Zhong } \\
\text { et al. (2017); Falloon (2017); Zhong, Wang e Chen (2016); } \\
\text { Pellas e Peroutseas (2016); Berland; Davis e Smith (2015); } \\
\text { Caglar et al. (2015); Mak (2014); Hu e Shepherd (2013); } \\
\text { González e Muñoz-Repiso (2017); Deitrick, Wilkerson e } \\
\text { Simoneau (2017). }\end{array}$ & $35,29 \%$ \\
\hline $\begin{array}{l}\text { Coleta e } \\
\text { Análise de } \\
\text { Dados }\end{array}$ & Hsu e Wang (2017). & $2,94 \%$ \\
\hline $\begin{array}{l}\text { Oficina ou } \\
\text { aula prática }\end{array}$ & $\begin{array}{l}\text { Papavlasopoulou (2017); Thieme (2017); Burns et al. } \\
\text { (2014); Wilkerson-Jerde (2014); Harlow e Leak (2014). }\end{array}$ & $14,7 \%$ \\
\hline $\begin{array}{l}\text { Jogar um } \\
\text { Game }\end{array}$ & $\begin{array}{l}\text { Apostolellis et al. (2014); Buffum (2016); Horn et al. } \\
\text { (2016). }\end{array}$ & $8,82 \%$ \\
\hline
\end{tabular}

\section{(F) Artefatos Criados}

Além das classes definidas anteriormente, notamos a necessidade de realizar uma classificação referente ao tipo de artefato trabalhado com alunos. O Pensamento Computacional envolve o desenvolvimento de algoritmos e projeto de sistemas para o mundo real, desta forma artefatos como jogos, simulações e robótica podem ser desenvolvidos durante as práticas propostas pelos trabalhos levantados com o objetivo de potencializar o aprendizado de conceitos do Pensamento Computacional. Portanto, as seguintes classes foram definidas: 
1. Jogos ou Animações Digitais: trabalhos que adotaram abordagens em que estudantes devem realizar o desenvolvimento de jogos ou animações digitais

2. Robótica: trabalhos em que estudantes devem utilizar, programar ou montar robôs/dispositivos robóticos.

3. Software - outros: estudos com abordagens em que estudantes devem desenvolver um software convencional, normalmente para ser aplicado em outras áreas do conhecimento.

4. Simulação: estudos que alunos deveriam montar uma simulação .

5. Coleção de dados: trabalhos em que estudantes deveriam montar uma base de dados ou um conjunto de informações de forma estruturada sobre um determinado assunto ou domínio.

6. Abstratos - algoritmos, lógica de programação ou modelos: trabalhos que não se encaixam nos itens anteriores onde, como finalidade, os alunos deveriam obter um algoritmo, uma solução para algum problema ou mesmo um modelo. Podemos ter, neste caso, artefatos mais abstratos.

7. Jogos e Robótica: trabalhos que se encaixam nas categorias 1 e 2.

Mais uma vez podemos evidenciar a necessidade de trabalhos com abordagens pautadas no uso de Kits de Robótica, apesar das inúmeras possibilidades que a Robótica Educacional pode proporcionar. Apenas três trabalhos focam no uso exclusivo da Robótica. Além disso, existe a necessidade de estudos que levam em consideração a produção de Simulações e Coleções de Dados.

Tabela 7: Tipos de Artefatos utilizados/desenvolvidos.

\begin{tabular}{|c|c|c|}
\hline Artefato & Trabalhos & Porcentagem \\
\hline $\begin{array}{l}\text { Jogos/Animaç } \\
\text { ões Digitais }\end{array}$ & $\begin{array}{c}\text { Thomas et al. (2017); Tabel et al. (2017); Fronza, Ioini e } \\
\text { Corral (2017); Gestwicki e Mcnely (2016); Reynolds (2016); } \\
\text { Hartl et al. (2015); Frydenberg (2015); Ke e Im (2014); Lee } \\
\text { (2016); Zhong et al. (2017); Falloon (2017); Zhong, Wang e } \\
\text { Chen (2016); Pellas e Peroutseas (2016); Mak (2014); Burns et } \\
\text { al. (2014); }\end{array}$ & $44,12 \%$ \\
\hline Robótica & $\begin{array}{l}\text { González e Muñoz-Repiso (2017); Damaševicius et al. (2017); } \\
\text { Fetaji et al. (2016). }\end{array}$ & $8,82 \%$ \\
\hline $\begin{array}{l}\text { Software } \\
\text { (outros) }\end{array}$ & $\begin{array}{l}\text { Pulimood, Pearson, Bates (2016); Goodman e Dekhtyar } \\
\text { (2014). }\end{array}$ & $5,88 \%$ \\
\hline Simulação & Caglar et al. (2015); Brady et al. (2017). & $5,88 \%$ \\
\hline $\begin{array}{l}\text { Abstratos - } \\
\text { algoritmos, } \\
\text { lógica de } \\
\text { programação } \\
\text { ou modelos }\end{array}$ & $\begin{array}{l}\text { Berland; Davis e Smith (2015); Hu e Shepherd (2013); Thieme } \\
\text { (2017); Silapachote e Srisuphab (2017); Deitrick, Wilkerson e } \\
\text { Simoneau (2017); Wilkerson-Jerde (2014); Buffum (2016); } \\
\text { Apostolellis et al. (2014); Horn et al. (2016); Harlow e Leak } \\
\text { (2014). }\end{array}$ & $29,41 \%$ \\
\hline $\begin{array}{l}\text { Coleção de } \\
\text { Dados }\end{array}$ & Hsu e Wang (2017). & $2,94 \%$ \\
\hline $\begin{array}{l}\text { Jogos e } \\
\text { Robótica }\end{array}$ & Papavlasopoulou (2017). & $2,94 \%$ \\
\hline
\end{tabular}




\section{(G) Tecnologias Utilizadas nas Atividades}

Como relatado anteriormente, os estudos podem incluir a utilização de ferramentas tecnológicas para que os alunos possam desenvolver as atividades propostas. Estas tecnologias podem envolver linguagens de programação, ambientes digitais de criação, jogos digitais, kits de robótica, jogos de tabuleiro entre outros. A Tabela 8 mostra a relação de ferramentas utilizadas nas atividades por trabalhos publicados. Estudos que utilizaram tecnologias menos populares, ou muito específicas, em suas atividades foram encaixados no item "Outros", como por exemplo, Deitrick, Wilkerson e Simoneau (2017) utilizaram a linguagem de programação R em suas atividades.

Tabela 8: Tipos de Artefatos utilizados/desenvolvidos.

\begin{tabular}{|c|c|c|}
\hline Tecnologia & Trabalhos & $\begin{array}{l}\text { Quantidade de } \\
\text { trabalhos }\end{array}$ \\
\hline Alice & $\begin{array}{l}\text { Zhong et al. (2017); Zhong, Wang e Chen (2016); Hartl et al. } \\
\text { (2015). }\end{array}$ & 3 \\
\hline AppInventor & Thomas et al. (2017). & 1 \\
\hline Arduino & Damaševicius et al. (2017). & 1 \\
\hline $\begin{array}{l}\text { Atividade lápis } \\
\text { papel }\end{array}$ & $\begin{array}{l}\text { Thomas et al. (2017); Fronza, Ioini e Corral (2017); } \\
\text { Silapachote e Srisuphab (2017). }\end{array}$ & 3 \\
\hline Bee-Bot & González e Muñoz-Repiso (2017). & 1 \\
\hline Construct & Frydenberg (2015). & 1 \\
\hline $\begin{array}{c}\text { Flash } \\
\text { (Actionscript) }\end{array}$ & Reynolds (2016). & 1 \\
\hline Google Forms & Hsu e Wang (2017). & 1 \\
\hline $\begin{array}{l}\text { Jogos } \\
\text { Analógicos }\end{array}$ & Apostolellis et al. (2014). & 1 \\
\hline $\begin{array}{l}\text { Lego } \\
\text { Mindstorms }\end{array}$ & $\begin{array}{l}\text { Mak (2014); Damaševicius et al. (2017); Gestwicki e Mcnely } \\
\text { (2016). }\end{array}$ & 3 \\
\hline Python & Hu e Shepherd (2013). & 1 \\
\hline $\begin{array}{l}\text { Scratch e } \\
\text { variantes. }\end{array}$ & $\begin{array}{c}\text { Thomas et al. (2017); Tabel et al. (2017); Fronza, Ioini e } \\
\text { Corral (2017); Falloon (2017); Pellas e Peroutseas (2016); Ke } \\
\text { e Im (2014); Mak (2014); Papavlasopoulou (2017); Burns et } \\
\text { al. (2014). }\end{array}$ & 9 \\
\hline Outros & $\begin{array}{l}\text { Silapachote e Srisuphab (2017); Brady et al. (2017); Gestwicki } \\
\text { e Mcnely (2016); Berland; Davis e Smith (2015); Caglar et al. } \\
\text { (2015); Goodman e Dekhtyar (2014); Wilkerson-Jerde (2014); } \\
\text { Deitrick, Wilkerson e Simoneau (2017); Thieme (2017); Lee } \\
\text { (2016); Pulimood, Pearson, Bates (2016); Horn et al. (2016); } \\
\text { Buffum (2016); Harlow e Leak (2014). }\end{array}$ & 14 \\
\hline
\end{tabular}

\section{(H) Modelo de Trabalho Conceitual}

Os trabalhos conceituais foram mapeados em três categorias: Ponto de Vista, Mapeamento Curricular e Revisão da Literatura como ilustra a Tabela 9. Além da escassez de trabalhos conceituais, podemos observar também a falta de estudos a respeito do mapeamento 
curricular no contexto da aprendizagem colaborativa, uma vez que não foram encontrados trabalhos relativos a esta categoria nas bases de dados da CAPES e ACM.

Tabela 9: Modelo de trabalho conceitual.

\begin{tabular}{|c|c|}
\hline Categoria & Trabalhos \\
\hline Ponto de Vista & Kafai (2016); Cortina (2015); \\
\hline $\begin{array}{c}\text { Mapeamento } \\
\text { Curricular }\end{array}$ & Não foram encontrados trabalhos nas bases de dados selecionadas. \\
\hline $\begin{array}{c}\text { Revisão da } \\
\text { Literatura }\end{array}$ & Kafai e Burke (2015). \\
\hline
\end{tabular}

\section{Discussão}

\subsection{Contexto Brasileiro}

Apesar de estudos brasileiros não terem aparecido como resultado nas bases de dados delimitadas pela pesquisa proposta, o cenário nacional a respeito de Aprendizagem Colaborativa aplicada ao desenvolvimento do Pensamento Computacional tem demostrado avanços consideráveis. Descreveremos a seguir alguns estudos significativos neste contexto.

Silva et al. (2018) apresentam um modelo gamificado para auxiliar o aprendizado de programação promovendo o engajamento estudantil e proporcionando um aprendizado personalizado. O estudo incluiu a avaliação dos indicadores de engajamento estudantil de Colaboração e Cooperação do modelo proposto. Bauer et al. (2017) relatam a respeito da experiência de um projeto de extensão. Este projeto foi voltado para o desenvolvimento do Pensamento Computacional em adolescentes por meio da programação de dispositivos móveis na plataforma AppInventor. A abordagem foi fundamentada em práticas colaborativas e aprendizagem baseada em problemas. Alves et al. (2016) apresentam uma unidade instrucional multidisciplinar envolvendo o Pensamento Computacional e a disciplina História e estudos sociais. Os autores descrevem um estudo de caso que incluiu o uso do ambiente Scratch e envolveu quatro turmas do quinto e sétimo anos do Ensino Fundamental. Os objetivos de aprendizagem da unidade instrucional proposta levaram em consideração criar, desenvolver, publicar e apresentar produtos utilizando recursos tecnológicos colaborativamente, programação em par entre outras atividades em grupo. Wangenheim et al. (2014) também propõem uma unidade instrucional que considerou, como objetivo de aprendizagem, o trabalho cooperativo e colaborativo entre colegas, professores e outros, usando tecnologia. No entanto, o objetivo dos autores foi o de descrever um estudo de caso do ensino de Computação com Scratch em turmas do ensino fundamental. O trabalho de França e Tedesco (2014) expõe um modelo colaborativo para o desenvolvimento do Pensamento Computacional aliado à autorregulação. $\mathrm{O}$ penC (modelo proposto) foi baseado em abordagens de resolução de problemas e envolve a colaboração no processo de avaliação em pares. O estudo não contempla experimentos práticos para verificar a eficácia na promoção das habilidades do Pensamento Computacional. Gärtner et al. (2012) apresentam uma abordagem fundamentada em metodologias ágeis. Os autores propõem uma ferramenta colaborativa de programação e descrevem um estudo de caso em que alunos de graduação utilizaram a ferramenta proposta para programar colaborativamente e aprender. 


\subsection{Conclusão}

Este artigo apresentou uma revisão sistemática da literatura a respeito de atividades de ensino para o desenvolvimento do Pensamento Computacional através da Aprendizagem Colaborativa. Para tal, foram realizadas buscas nas bases de dados de periódicos da CAPES e da ACM. Os resultados mostraram avanços e lacunas encontradas no estado da arte. Dentre os avanços, podemos destacar o aumento de produções com o passar do tempo, a quantidade de trabalhos que exploram a utilização ou produção de Jogos Digitais, a quantidade de trabalhos que focam em atividades de resolução de séries de desafios, a exploração de técnicas de colaboração inspiradas no desenvolvimento de projetos e na programação em par. Como lacunas, podemos observar a carência de trabalhos de cunho conceitual e a necessidade de mais estudos que exploram o desenvolvimento colaborativo de projetos de robótica. Outra lacuna que fica bastante evidente é a deficiência de estudos que exploram outras abordagens ou métodos de colaboração mais estruturados. A maioria dos trabalhos utiliza uma abordagem não estruturada, em que a colaboração parece ficar sob a autonomia do grupo de estudantes, não ficando claro como as interações sociais entre alunos irão ocorrer de fato. Faz-se necessário a realização de estudos que exploram outras estratégias mais claras de Aprendizagem Colaborativa, incluindo métodos normalmente utilizados para ensino de programação, como o Dojo de Programação ou atividades de competições entre equipes, para se entender como os processos colaborativos podem se manifestar neste meio. Pode-se observar, ainda, a necessidade de trabalhos dedicados a docentes, sendo que apenas dois trabalhos relataram ter realizado atividades com este público.

Sobre a questão de pesquisa, que guiou este estudo, podemos perceber que a Aprendizagem Colaborativa tem sido utilizada através das classes encontradas nos modelos de colaboração (Tabela 5), sendo que as três abordagens mais utilizadas foram: Trabalho em Grupo, Solução Colaborativa de Série de Problemas e Programação em Par. Sendo assim, na maioria das vezes, as atividades ditas colaborativas se manifestam através de trabalhos em grupos "livres", porém, nota-se também uma influência das metodologias ágeis nas aboardagens, uma vez que a programação por pares se encontra entre as estratégias mais utilizadas. Ainda sobre a questão de pesquisa, as categorias encontradas no item Tipos de Atividades Realizadas (Tabela 6), assim como no item Artefatos Utilizados/Desenvolvidos (Tabela 7), indicam quais atividades relacionadas ao Pensamento Computacional tem sido trabalhadas colaborativamente. Entre as principais atividades realizadas estão: o Projeto de Desenvolvimento de Jogos e Animações, Solução de Desafios Propostos e Jogar um Jogo. Observa-se desta forma uma influência dos jogos (tanto no uso quanto no desenvolvimento) nas atividades colaborativas. Isto mostra evidências de que a questão lúdica pode ser um fator positivo para que ocorra colaboração no aprendizado do Pensamento Computacional. Cabe observar que neste artigo apenas foram encontradas algumas evidências a respeito de como a Aprendizagem Colaborativa tem se manifestado em atividades que estimulam o Pensamento Computacional em algumas das principais pesquisas publicadas na literatura. Uma resposta mais conclusiva para esta questão exigiria a realização de novos estudos conceituais e empíricas que fogem do escopo desta pesquisa.

Este trabalho revela que muito ainda precisa ser feito para a compreensão do uso do Aprendizado Colaborativo para dar suporte ao desenvolvimento do Pensamento Computacional em estudantes. $\mathrm{O}$ ascendente número de estudos a respeito deste tema representa um fato otimista e uma tendência consolidada para contribuir para o ensino de forma geral.

\section{Referências}


ACM. (2017). ACM Digital Library. [online] Disponível em: https://dl.acm.org/dl.cfm/ [Acessado 2 Out. 2017].

Alves, N., da C.; Rodrigues, P. E.; Borgatto, A. F.; Wangenheim, C. G. von \& Hauck, J. C. R. (2016). Ensino de Computação de Forma Multidisciplinar em Disciplinas de História no Ensino Fundamental - Um Estudo de Caso. Brazilian Journal of Computers in Education (Revista Brasileira de Informática na Educação - RBIE), 24(3), 31-46. doi:10.5753/rbie.2016.24.3.31. [GS Search]

Apostolellis, P.; Stewart, M.; Frisina, C. \& Kafura, D. (2014, Junho). RaBit EscAPE: a board game for computational thinking. Proceedings of the conference on Interaction design and children, Aarhus, Denmark. doi:10.1145/2593968.2610489. [GS Search]

Bauer, R. D.; Flores, G. L. M. \& Crestani, Â. N. V. (2017, Outubro) Projeto codIFic@r: Oficinas de Programação em Dispositivos Móveis no Ensino Fundamental. In Workshops of Brazilian Symposium on Computers in Education (Workshops do Congresso Brasileiro de Informática na Educação - WCBIE) (Vol. 6, No. 1, p. 1210-1219). doi: 10.5753/cbie.wcbie.2017.1210. [GS Search]

Berland, M.; Davis, D. \& Smith, C. (2015). AMOEBA: Designing for collaboration in computer science classrooms through live learning analytics. International Journal of ComputerSupported Collaborative Learning, 10(4), 425-447. doi:10.1007/s11412-015-9217-z. [GS Search]

Barcelos, T. S. (2014), Relações entre o pensamento computacional e a matemática em atividades didáticas de construção de jogos digitais. 2014. Tese de Doutorado em Ensino de Ciências e Matemática, Universidade Cruzeiro do Sul. São Paulo, Brasil. 276 p. [GS $\underline{\text { Search }}$

Barr, V. \& Stephenson, C. (2011) Bringing computational thinking to K-12. ACM Inroads 2, 1, 48-54. doi:10.1145/1929887.1929905. [GS Search]

Brady, C.; Orton, K.; Weintrop, D.; Anton, G.; Rodriguez, S. \& Wilensky, U. (2017). All Roads Lead to Computing: Making, Participatory Simulations, and Social Computing as Pathways to Computer Science. IEEE Transactions on Education, 60(1), 59-66. doi:0.1109/TE.2016.2622680. [GS Search]

Buffum, P. S.; Frankosky, M.; Boyer, K. E.; Wiebe, E. N.; Mott, B. W.; Lester, J. C. (2016). Collaboration and Gender Equity in Game-Based Learning for Middle School Computer Science. Computing in Science \& Engineering, 18(2), 18-28. doi:10.1109/MCSE.2016.37. [GS Search]

Burns, R.; Eugene, W.; Barnes, T.; Chandler, S.; Harwell, M. \& Omokaro, O. (2014). Reflections from a Computational Service Learning Trip to Haiti. Journal of Computing Sciences in Colleges. 29 (3). [GS Search]

Caglar, F.; Shekhar, S.; Gokhale, A.; Basu, S.; Rafi, T.; Kinnebrew, J. \& Biswas, G. (2015). Cloud-hosted simulation-as-a-service for high school STEM education. Simulation Modelling Practice and Theory, 58, 255-273. doi:10.1016/j.simpat.2015.06.006. [GS Search]

CAPES. (2017). Portal Periodicos CAPES. [online] Disponível em: http://www.periodicos.capes.gov.br/ [Acessado 2 Out. 2017].

Cortina, T. (2015). Reaching a Broader Population of Students Through "Unplugged" Activities. Communications of the ACM, 58(3), 25-27. doi:10.1145/2723671. [GS Search] 
CSTA\&ISTE (2011). Computational Thinking Leadership Toolkit. [online] Disponível em: http://csta.acm.org/curriculum/sub/compthinking.html [Acesso 10 Out. 2017].

Damaševicius, R.; Narbutaite, L.; Plauska, I. \& Blažauskas, T. (2017) Advances in the Use of Educational Robots in Project-Based Teaching. TEM Journal, 6(2), 342-348. doi:10.18421/TEM62-20. [GS Search]

Deitrick, E.; Wilkerson, M. H. \& Simoneau, E. (2017, Agosto). Understanding Student Collaboration in Interdisciplinary Computing Activities. Proceedings of the 2017 ACM Conference on International Computing Education Research (ICER '17), Tacoma, WA, USA. doi:10.1145/3105726.3106193. [GS Search]

Dillenbourg, P. (1999). What do you mean by collaborative learning?. Collaborativelearning: Cognitive and Computational Approaches. Oxford: Elsevier, 1-19. doi:10.1016/S03601315(00)00011-7. [GS Search]

Falloon, G. (2016). An Analysis of Young Students' Thinking when Completing Basic Coding Tasks Using Scratch Jnr. On the iPad. Journal of Computer Assisted Learning, 32, 576593. doi:10.1111/jcal.12155. [GS Search]

Fetaji, M.; Fetaji, B.; Sukic, C.; Gylcan, A. \& Ebibi, Mi. (2016). Case Study Analyses of the Impact of Flipped Learning in Teaching Programming Robots. TEM Journal, 5(4), 560565. doi:10.18421/TEM54-21. [GS Search]

França, R. S. de \& Tedesco, P. C. de A. R. (2014, Novembro) Um Modelo Colaborativo para a Aprendizagem do Pensamento Computacional aliado à Autorregulação. In Brazilian Symposium on Computers in Education (Simpósio Brasileiro de Informática na EducaçãoSBIE) (Vol. 25, No. 1, p. 1133-1142). doi: 10.5753/cbie.sbie.2014.1133. [GS Search]

Fronza, I. \& Ioini, N. \& Corral, L. (2017) Teaching Computational Thinking Using Agile Software Engineering Methods: A Framework for Middle Schools. ACM Transactions on Computing Education (TOCE), 17(4), 1-28. doi:10.1145/3055258. [GS Search]

Frydenberg, M. (2015). Achieving Digital Literacy through Game Development: an authentic learning experience. Interactive Technology and Smart Education, 12(4), 256-269. doi:10.1108/ITSE-08-2015-0022. [GS Search]

Gärtner, V. C.; Pinto, S. C. C. S. \& Gómez A. T (2012). Facilitando o aprendizado de programação sob a ótica de metodologias ágeis. Brazilian Journal of Computers in Education (Revista Brasileira de Informática na Educação - RBIE), 20 (2), 54-68. doi:10.5753/rbie.2012.20.2.54. [GS Search]

Gestwicki, P. \& Mcnely, B. (2016). Interdisciplinary Projects in the Academic Studio. ACM Transactions on Computing Education (TOCE), 16(2), 1-24. doi:10.1145/2732157. [GS Search]

Gokhale, A. A. (1995) Collaborative Learning Enhances Critical Thinking. Journal of Technology Education, 7 (1). doi:10.1007/978-1-4419-1428-6_910. [GS Search]

González, Y. A. C. \& Muñoz-Repiso, A. G.-V. (2017, Outubro) Development of Computational Thinking and Collaborative Learning in Kindergarten using Programmable Educational Robots: a teacher training experience. Proceedings of the 5th International Conference on Technological Ecosystems for Enhancing Multiculturality (TEEM 2017), Cádiz, Espanha. doi: $10.1145 / 3144826.3145353$. [GS Search] 
Goodman, A. L. \& Dekhtyar, A. (2014) Teaching bioinformatics in concert. PLoS computational biology, 10(11). doi: 10.1371/journal.pcbi.1003896. doi:10.1371/journal.pcbi.1003896. [GS Search]

Harlow, D. B. \& Leak, A. E. (2014). Mapping students' ideas to understand learning in a collaborative programming environment. Computer Science Education, 24(2-3), 229-247. doi:10.1080/08993408.2014.963360. [GS Search]

Hartl, A. C.; Delay, D; Laursen, B.; Denner, J.; Werner, L.; Campe, S. \& Ortiz, E. (2015) Dyadic Instruction for Middle School Students: Liking promotes learning. Learning and Individual Differences, 44, 33-39. doi:10.1016/j.lindif.2015.11.002. [GS Search]

Horn, B.; Clark, C.; Strom, O.; Chao, H.; Stahl, A. J.; Harteveld, C. \& Smith, G. Design Insights into the Creation and Evaluation of a Computer Science Educational Game. (2016, Fevereiro). Proceedings of the 47th ACM Technical Symposium on Computing Science Education (SIGCSE '16), Memphis, TN, USA. doi:10.1145/2839509.2844656. [GS Search]

Hsu, H.-Y. \& Wang, S.-. (2017). Using Google Forms to Collect and Analyze Data. Integrating technology. Science Scope, 40(8), 64-67. doi:10.2505/4/ss17_040_08_64. [GS Search]

Hu, H. \& Shepherd, T. (2013) Using POGIL to help students learn to program. ACM Transactions on Computing Education (TOCE), 13(3), 1-23. doi:10.1145/2499947.2499950. [GS Search]

Kafai, Y. B. (2016). From Computational Thinking to Computational Participation in K12 Education. Communications of the ACM, 59(8), 26-27. doi:10.1145/2955114. [GS Search]

Kafai Y. B. \& Burke, Q. (2015) Constructionist Gaming: Understanding the Benefits of Making Games for Learning. Educational Psychologist, 50(4), 313-334. doi:10.1080/00461520.2015.1124022. [GS Search]

Ke, F. \& Im, T. A. (2014). Case Study on Collective Cognition and Operation in Team-Based Computer Game Design by Middle-School Children. International Journal of Technology and Design Education, 24(2), 187-201. doi:10.1007/s10798-013-9248-6. [GS Search]

Laal, M. \& Laal, M. (2012) Collaborative learning: what is it? Procedia - Social and Behavioral Sciences, 31, 491 - 495. doi:10.1016/j.sbspro.2011.12.092. [GS Search]

Lee, K. T. (2016, Novembro) Use of Tangible Learning in Stem Education. SIGGRAPH ASIA 2016 Mobile Graphics and Interactive Applications, Macau, China. doi:10.1145/2999508.3008582. [GS Search]

Mak, J. (2014) Coding in the Elementary School Classroom. Learning \& Leading with Technology, 41(6), 26-28. [GS Search]

Moreira, M. A. (2011). Aprendizagem Significativa: um conceito subjacente (Meaningful Learning: an underlying concept). Aprendizagem Significativa em Revista/Meaningful Learning Review - 1(3), 25-46. [GS Search]

Panitiz, T. (1999). Collaborative versus Cooperative Learning: A comparison of two concepts wich will help us understand the underlying nature of interactive learning. Educational Resources Information Center ERIC. [GS Search]

Papavlasopoulou, S.; Sharma, K.; Giannakos, M. \& Jaccheri, L. (2017, Junho). Using EyeTracking to Unveil Differences Between Kids and Teens in Coding Activities. Proceedings of the 2017 Conference on Interaction Design and Children, Stanford, California, USA. doi: $10.1145 / 3078072.3079740$. [GS Search] 
Papert, S. (1980) Mindstorms: Children, Computers and Powerful Ideas. Basic Books. doi:10.1037/h0098915. [GS Search]

Papert, S. (1993) The children's machine. Manchester: Technology Review. doi:10.1207/s15327809jls0604_4. [GS Search]

Pellas, N. \& Peroutseas, E. (2016). Gaming in Second Life via Scratch4SL: Engaging High School Students in Programming Courses. Journal of Educational Computing Research, 54(1), 108-143. doi:10.1177/0735633115612785. [GS Search]

Pulimood, S. M.; Pearson, K.; Bates, D. C. A. (2016, Fevereiro). Study on the Impact of Multidisciplinary Collaboration on Computational Thinking. Proceedings of the 47th ACM Technical Symposium on Computing Science Education, Memphis, Tennessee, USA. doi:10.1145/2839509.2844636. [GS Search]

Reynolds, R. B. (2016). Relationships Among Tasks, Collaborative Inquiry Processes, Inquiry Resolutions, and Knowledge Outcomes in Adolescents During Guided Discovery-Based Game Design in School. Journal of Information Science, 42(1), 35-58. doi:10.1177/0165551515614537. [GS Search]

Sato, D. T., Corbucci, H., e Bravo, M. V. (2008, Agosto) Coding dojo: An environment for learning and sharing agile practices. Proceedings of Agile Conference (AGILE'08), Toronto, ON, Canada. doi:10.1109/Agile.2008.1. [GS Search]

Silapachote, P. \& Srisuphab, A. (2017) Engineering Courses on Computational Thinking Through Solving Problems in Artificial Intelligence. International Journal of Engineering Pedagogy (iJEP), 7(3), 34-49. doi:10.3991/ijep.v7i3.6951. [GS Search]

Silva, T. S. C., Melo, J. C. B. \& Tedesco, P. C. A. R. (2018). A Model to Promote Student Engagement in Programming Learning Using Gamification (Um Modelo para Promover o Engajamento Estudantil no Aprendizado de Programação Utilizando Gamification). Brazilian Journal of Computers in Education (Revista Brasileira de Informática na Educação - RBIE), 26(3), 120-138. doi: 10.5753/RBIE.2018.26.03.120. [GS Search]

Smith, B.L., e Macgregor, J. (1992). Collaborative Learning: A Sourcebook for Higher Education. University Park, PA: National Center on Postsecondary Teaching, Learning, and Assessment (NCTLA). 9-22. [GS Search]

Tabel, O.; Jensen, J.; Dybdal, M. \& Bjørn, P. (2017). Coding as a Social and Tangible Activity. Interactions, 24(6), 70-73. doi:10.1145/3137099. [GS Search]

Thieme, A.; Morrison, C.; Villar, N.; Grayson, M. \& Lindley, S. (2017, Junho). Enabling Collaboration in Learning Computer Programing Inclusive of Children with Vision Impairments. Proceedings of the Conference on Designing Interactive Systems (DIS '17), Edinburgh, United Kingdom. doi:10.1145/3064663.3064689. [GS Search]

Thomas, J.; Rankin, Y.; Minor, R. \& Sun, L. (2017). Exploring the Difficulties AfricanAmerican Middle School Girls Face Enacting Computational Algorithmic Thinking over three Years while Designing Games for Social Change. Computer Supported Cooperative Work (CSCW), 26(4), 389-421. doi:10.1007/s10606-017-9292-y. [GS Search]

Wangenheim, C. G. von; Nunes, V. R. \& Santos, G. D. dos (2014) Ensino de Computação com SCRATCH no Ensino Fundamental - Um Estudo de Caso. Brazilian Journal of Computers in Education (Revista Brasileira de Informática na Educação - RBIE), 22 (3). doi: 10.5753/RBIE.2014.22.03.115. doi:10.5753/RBIE.2014.22.03.115. [GS Search] 
Wilkerson-Jerde, M. (2014) Construction, Categorization, and Consensus: student generated computational artifacts as a context for disciplinary reflection. Educational Technology Research and Development, 62(1), 99-121. doi:10.1007/s11423-013-9327-0. [GS Search]

Wing, J. M. (2006). Computational thinking. Communications of ACM, 49(3): 33-35. [GS Search]

Zhong, B.; Wang, Q. \& Chen, J. (2016) The Impact of Social Factors on Pair Programming in a Primary School. Computers in Human Behavior, 64, 423-431. doi:10.1016/j.chb.2016.07.017. [GS Search]

Zhong, B.; Wang, Q.; Chen, J. \& Li, Y. (2017) Investigating the Period of Switching Roles in Pair Programming in a Primary School. Educational Technology \& Society, 20(3), 220-233. [GS Search] 